\title{
Fehlende hämodynamische Antwort bei erhaltener neuronaler Funktion: Pitfall der funktionellen MRT bei Patienten mit stenosierenden Gefäßprozessen
}

Die funktionelle Magnetresonanztomographie (fMRT) erlaubt es, regionale Änderungen der zerebralen Durchblutung infolge neuronaler Aktivierungen nicht-invasiv darzustellen. Daher wird die fMRT zunehmend auch klinisch eingesetzt, insbesondere zur Lokalisation motorischer und sprachrelevanter Hirnareale. In mehreren Studien wurde eine weitgehende Übereinstimmung der Ergebnisse von fMRT und Wada-Test (seitengetrennte intrakarotidale AmytalInjektionen) bei der Bestimmung der Hemisphärendominanz für sprachliche Funktionen gezeigt (u.a. Desmond, JE et al., Brain 1995, 118: 1411). Das Ausmaß der Hemisphärenspezialisierung kann durch Berechnung von Lateralisationsindizes aus fMRT-Untersuchungen quantifiziert werden (Binder, JR et al., Neurology 1996; 46: 978). Wir berichten über einen Patienten mit linksseitigem inkomplettem Verschluß der A.cerebri media, bei dem trotz erhaltener neuronaler Funktion kein BOLD-Effekt nachzuweisen war.

\section{Fallbeschreibung}

Der 62jährige Patient wurde durch rezidivierende Konzentrations- und apraktische Störungen auffällig. Bei der dopplersonographischen Untersuchung zeigte sich ein inkompletter Verschluß der A. cerebri media links, der angiographisch bestätigt wurde. Das linke Mediaterritorium wurde antegrad lediglich über einen erhaltenen Mediaast und retrograd über leptomeningeale Kollateralen aus der A. cerebri anterior versorgt (Abb.1). Eine kraniale Computertomographie erbrachte keinen pathologischen Befund. Die strukturelle MRTBildgebung zeigte in $\mathrm{T}_{2}$-Gewichtung kleinfleckige Signalanhebungen in den Stammganglien und im frontalen und parietalen Marklager links ohne Diffusionsstörung, die als ältere ischämische Läsionen gedeutet wurden. Kortikale Defekte lagen nicht vor. Eine Hirnszintigraphie mittels ${ }^{99 \mathrm{~m} T c}$ SPECT zeigte bereits in Ruhe eine verminderte Anreicherung des Perfusionstracers im Stromgebiet der linken A.cerebri media mit deutlicher Zunahme des Perfusionsdefizits nach Infusion von Acetazolamid. 


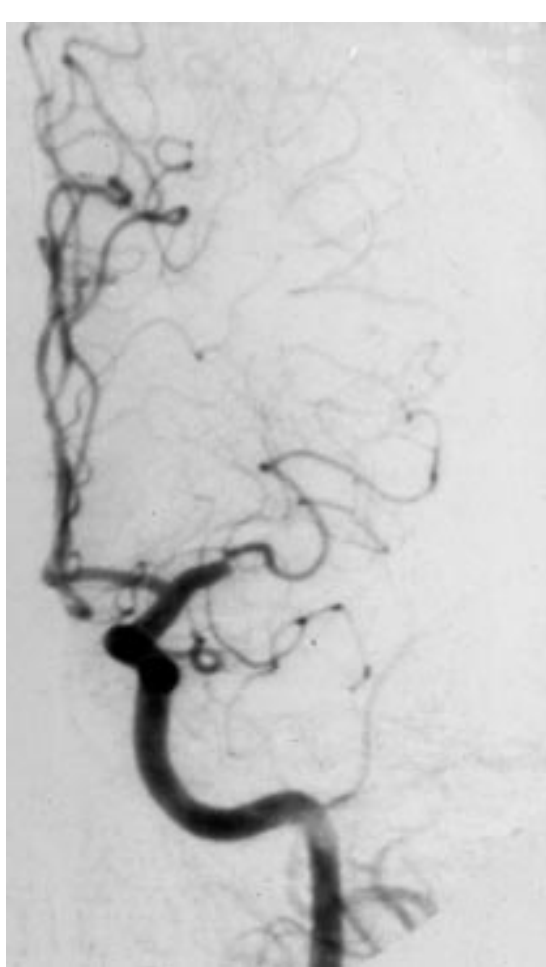

Abb.1 Intraarterielle DSA der linken ASC. Verschluß der A. cerebri media an der Bifurkation bei lediglich einem erhaltenen Mediahauptast. Leptomeningeale Kollateralen zum Mediaareal aus der A. cerebri anterior.

Die stationäre Aufnahme erfolgte, um die Indikation zur Anlage eines extra-intrakraniellen Bypasses $z u$ prüfen. Der neurologische Befund war bis auf eine distal betonte sensomotorische Paraparese der Beine im Rahmen einer langjährigen Polyneuropathie unauffällig, insbesondere bestanden keine neurologischen Defizite an den oberen Extremi- täten. Bei der neuropsychologischen Untersuchung zeigte sich ein inkomplettes Gerstmann-Syndrom (Dyskalkulie, Rechts-Linksschwäche, Dysgraphie). Daneben bestand eine geringe Dyslexie. Die Spontansprache war weitgehend unauffällig, nach längeren Belastungssituationen traten vereinzelte Paraphasien auf.

Der Patient gab an, Linkshänder zu sein, die neuropsychologische Händigkeitsuntersuchung erbrachte eine Beidhändigkeit. Sprachliche Funktionen sind bei Beidhändern variabel lateralisiert und häufig bilateral repräsentiert (Kuthen, M; Fortschr. Neurol. Psychiat. 1993, 61: 77). Der Patient wurde zur MRT überwiesen, um die zerebrale Sprachrepräsentation zu ermitteln und so das Risiko des Auftretens einer kompletten Aphasie besser abschätzen zu können.

Die Untersuchung erfolgte an einem 1,5T Scanner mit zirkulär polarisierter Kopfspule. Für die funktionellen Messungen wurden Mehrschicht-Gradienten-Echo EPI (Echoplanar imaging) Sequenzen verwendet (TR 1,76 ms pro Phasenkodierschritt, TE $84 \mathrm{~ms}$, FoV 256 mm, Matrix $128 \times 128)$. Es wurde ein Wortgenerierungsparadigma (innerliches $\mathrm{Ge}$ nerieren von Verben zu akustisch vorgegebenen Nomina) (FitzGerald, DB et al.; AJNR 1997; 18: 1529) mit alternierenden Aktivierungs- und Kontrollbedingungen (Entspannen) (je 8 Blocks à 5 Messungen) durchgeführt. Zusätzlich wurde ein motorisches Paradigma (bilateraler Faustschluß versus Ruhe) durchgeführt. Beide Aufgaben wurden zunächst außerhalb des Scanners geübt und vom Pa- tienten problemlos bewältigt. Die statistische Auswertung erfolgte mit der Software BrainVoyager ${ }^{\circledR}$ und umfaßte eine Bewegungskorrektur, räumliche und zeitliche Glättung der Daten sowie eine Korrelationsanalyse. Es wurden mehrere Berechnungen mit Verschiebung der Boxcar-Referenzkurve um 1, 2 und 3 Messungen gegenüber den Stimulationsphasen durchgeführt.

Bei dem Sprachparadigma zeigte sich eine Aktivierung im frontalen Operkulum rechts und im Gyrus temporalis superior rechts, links frontal und temporal bestanden auch bei niedrigen Schwellenwerten keine signifikanten Signaldifferenzen zwischen Aktivierungsund Kontrollbedingungen (Abb. 2). Bei dem motorischen Paradigma fand sich, obwohl bilateral synchrone Handbewegungen ausgeführt wurden, ausschließlich rechtshemisphärisch eine Aktivierung des primären motosensorischen Handareals (Abb.3). Auch bei Verschiebung der Referenzkurve konnte keine signifikante Aktivierung linkshemisphärischer Sprachareale bzw. des linkshemisphärischen Motorcortex nachgewiesen werden.

\section{Ergebnisse}

Grundlage der fMRT ist der sogenannte BOLD-(blood oxygen level dependent) Effekt, der darauf beruht, daß der vermehrte Sauerstoffbedarf aktiver Nervenzellverbände physiologisch durch eine regionale Steigerung der Hirndurchblutung überkompensiert wird. Der relative Abfall von Desoxy-Hämoglobin bewirkt einen Signalanstieg in suszeptibilitäts-
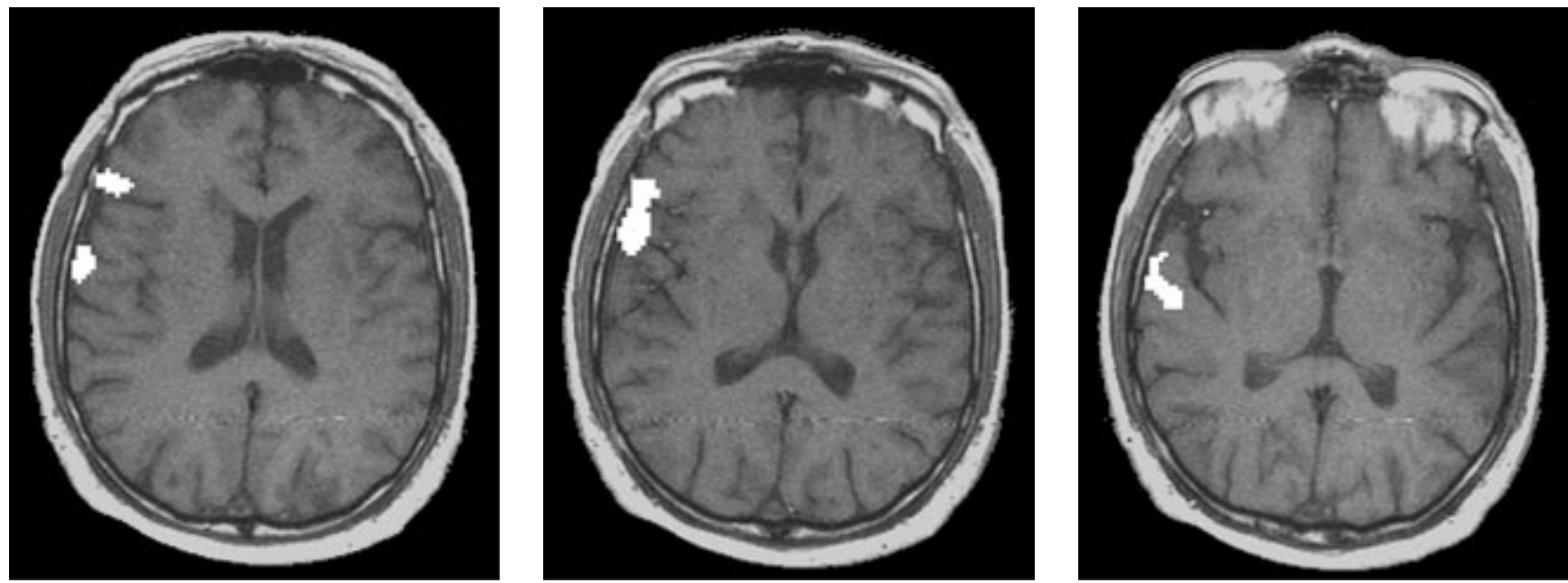

Abb.2 Sprachliche fMRT: innerliches Generieren von Verben zu akustisch vorgegebenen Nomina. Rechtsseitige Aktivierung im frontalen Operkulum und Gyrus temporalis superior. Korrelationsanalyse, Pixel mit $r>0.4$ weiß kodiert. 
a, b
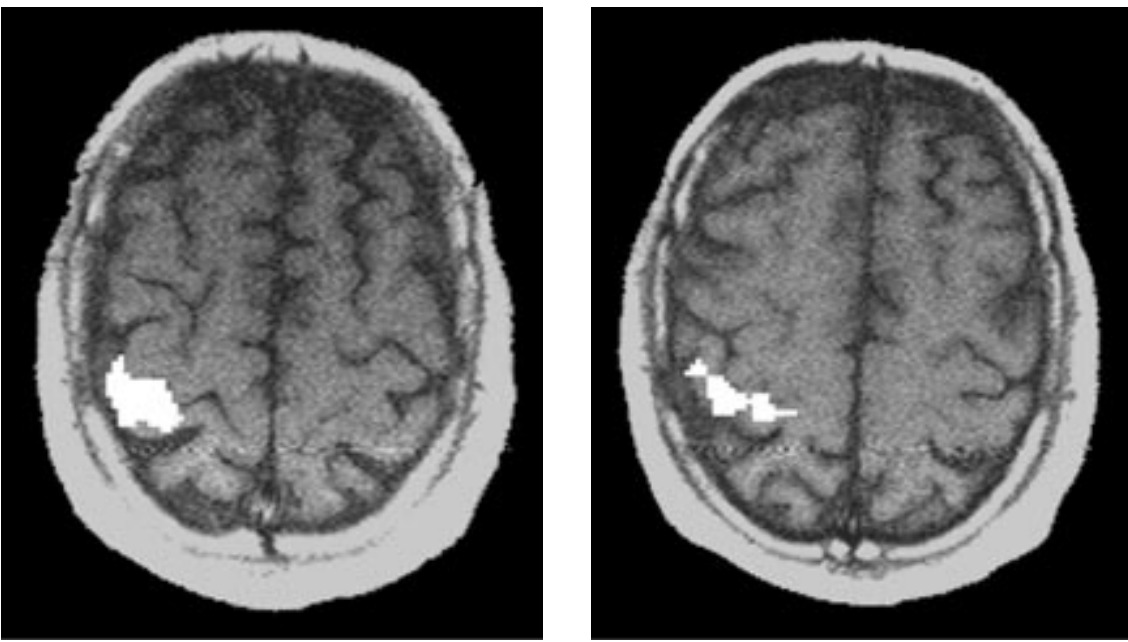

Abb. 3 Motorische fMRT: bilateraler Faustschluß. Ausschließlich rechtsseitige Aktivierung des primären motosensorischen Handareals. Korrelationsanalyse, Pixel mit $r>0.4$ weiß kodiert.

sensitiven MR-Sequenzen (Kwong KK et al; Proc. Natl. Acad. Sci. 1992: 89: 5675).

Bei dem hier vorgestellten Patienten mit chronischem inkomplettem linksseitigem Mediaverschluß fand sich in der fMRT weder bei der motorischen noch bei der sprachlichen Aufgabe ein BOLDEffekt im linken Mediaareal, obwohl zum Untersuchungszeitpunkt weder eine Parese noch eine manifeste Sprachstörung vorlagen. Hingegen zeigten sich rechtshemisphärisch plausible Aktivierungsmuster sowohl bei der motorischen als auch bei der Sprachaufgabe. Die ausschließlich rechtsseitige BOLD-Antwort bei dem Sprachparadigma beweist in diesem Fall nicht, daß eine - prinzipiell mögliche - exklusiv rechtsseitige Sprachrepräsentation oder rechtshemisphärische Sprachdominanz besteht. Das Vorliegen eines inkompletten Gerstmann-Syndroms und - wenn auch geringer - aphasischer Störungen spricht vielmehr dafür, daß die linke Hemisphäre bei sprachlichen Funktionen zumindest mitbeteiligt und möglicherweise sogar die dominante ist. Da auch bei bilateralen Handbewegungen keine hämodynamische Antwort in der linken Hemisphäre auftrat, ist zu vermuten, daß mit der Sprachaufgabe assoziierte linkshemisphärische neuronale Aktivierungen der Darstellung in der MRT entgangen sind. Die Berechnung eines Lateralisationsindex aus der Anzahl überschwelliger Voxel in beiden Hemisphären ist in diesem Falle also problematisch und könnte irreführende Ergebnisse erbringen.
In der motorischen fMRT wurde bei unserem Patienten lediglich ein bilaterales Faustschlußparadigma durchgeführt. Eine seitengetrennte motorische Untersuchung hätte interessante Aufschlüsse über ipsilaterale Aktivierungen bei Bewegungen der rechten Hand erbringen können, die bei dem durchgeführten bilateralen Paradigma maskiert wurden.

Da das linke Mediaterritorium retrograd über leptomeningeale Kollateralen aus der A.cerebri anterior versorgt wurde, wäre es vorstellbar, daß der BOLD-Effekt zeitlich verzögert auftrat. Ein solcher zeitlicher Versatz der hämodynamischen Antwort wurde durch Verschiebung der Referenzkurve der Korrelationsanalyse ausgeschlossen.

Eine lokale Durchblutungssteigerung ist nur dann möglich, wenn nicht bereits unter Ruhebedingungen eine maximale Vasodilatation vorliegt. Stenosen extraoder intrazerebraler hirnversorgender Gefäße können durch Vasodilatation der nachgeschalteten Arteriolen lange kompensiert werden. Bei unserem Patienten ist offensichtlich die Reservekapazität im Mediastromgebiet links so stark eingeschränkt, daß die neuronalen Aktivierungen keine mittels fMRT detektierbare lokale hämodynamische Antwort mehr bewirken können. Da elektrische neuronale Aktivität Sauerstoff erfordert, muß davon ausgegangen werden, daß die regionale Sauerstoffextraktion erhöht ist und somit die lokale Konzentration von paramagnetischem Desoxy-Hämoglobin ansteigt. Bei dieser
Konstellation wäre ein mit den Aufgaben korrelierter Signalabfall vorstellbar. Einen solchen „negativen BOLD-Effekt“ konnten wir jedoch nicht nachweisen.

Die Abhängigkeit des BOLD-Effektes von der zerebralen Reservekapazität muß bei der klinischen Anwendung der fMRT, insbesondere bei Untersuchungen der Lateralisierung zerebraler Funktionen, berücksichtigt werden. Um Fehlinterpretationen $\mathrm{zu}$ vermeiden, sollten bei fMRT-Untersuchungen hämodynamisch relevante Gefäßstenosen ausgeschlossen werden.

Joachim Spreer, Freiburg; H. Yahya, Frankfurt/M.; P. Raab, Frankfurt/M. 